\title{
Placebo controlled trial of fusidic acid gel and oxytetracycline for recurrent blepharitis and
} rosacea

\author{
D V Seal, P Wright, L Ficker, K Hagan, M Troski, P Menday
}

\begin{abstract}
A prospective, randomised, double blind, partial crossover, placebo controlled trial has been conducted to compare the performance of topical fusidic acid gel (Fucithalmic) and oral oxytetracycline as treatment for symptomatic chronic blepharitis. Treatment success was judged both by a reduction in symptoms and clinical examination before and after therapy. Seventy five per cent of patients with blepharitis and associated rosacea were symptomatically improved by fusidic acid gel and $50 \%$ by oxytetracycline, but fewer $(35 \%)$ appeared to benefit from the combination. Patients with chronic blepharitis of other aetiologies did not respond to fusidic acid gel but $25 \%$ did benefit from oxytetracycline and $30 \%$ from the combination. Our results demonstrate the need to investigate patients with blepharitis for concomitant rosacea as they respond well to targeted therapy.

(Brf Ophthalmol 1995; 79: 42-45)
\end{abstract}

Chronic blepharitis is a common problem but is difficult to manage because various aetiologies are involved. Universal treatment cannot therefore be applied and, until now, specific therapy has not been identified for each subgroup. Symptomatic relief remains the major therapeutic contribution.

There is controversy over the role of inflammation as distinct from infection, but in some patients the two may be interrelated. Seborrhoeic disease and meibomian dysfunction are considered primarily inflammatory. ${ }^{1}$ Atopic keratoconjunctivitis, characterised by heavy Staphylococcus aureus colonisation, appears inflammatory rather than infective. Folliculitis and ulcerative blepharitis in 'staphylococcal' lid disease usually represent infection caused by $S$ aureus which may be complicated by cell mediated marginal keratitis. ${ }^{2} 3$

We have demonstrated enhanced cell mediated immunity (CMI) to $S$ aureus, but not to coagulase negative staphylococci (CNS), among patients with symptomatic blepharitis. We have found that coagulase negative staphylococci represent normal commensal flora. ${ }^{24}$ The pathogenic role of bacteria isolated from patients with chronic blepharitis remains controversial and it is not surprising that there is continued debate over its treatment. Therapeutic approaches have included lid hygiene, topical and systemic anti-staphylococcal antibiotics in addition to anti-inflammatory therapy.

Fusidic acid has been in clinical use since 1962 and is particularly effective against staphylococci. It has a steroid structure but no glucocorticoid activity. A new gel preparation containing $1 \%$ microcrystalline fusidic acid (Fucithalmic) has recently been shown to be effective for treating acute bacterial conjunctivitis and for reducing the staphylococcal lid flora before surgery. ${ }^{5-8}$

Oxytetracycline has been selected for the treatment of patients with chronic blepharitis as it has both anti-inflammatory and antistaphylococcal properties. ${ }^{910}$ It has been demonstrated to be effective in a controlled trial in ocular rosacea for corneal infiltrates and conjunctival hyperaemia ${ }^{11}$ and in non-ocular rosacea for papular disease. ${ }^{12}$ These beneficial effects have also been demonstrated with doxycycline. ${ }^{13}$

We have studied the subjective and objective responses of patients with chronic blepharitis of various aetiologies treated with fusidic acid and oxytetracycline in a randomised, controlled prospective way.

\section{Materials and methods}

Patients suffering from chronic blepharitis were offered the opportunity to participate in this trial. The majority had taken part in a previous study, assessing the role of staphylococcal CMI in this condition, when their symptoms and signs were tabulated in detail. ${ }^{2}$ This study was designed as a prospective, randomised, double blind, placebo controlled, partial crossover group comparison trial of the effect of fusidic acid and oxytetracycline to give symptomatic relief. A similar protocol for assessing symptoms and signs was used as before. ${ }^{2}$ Informed, written consent was obtained from all patients with chronic blepharitis entering the study. Exclusion criteria were known hypersensitivity to fusidic acid, oxytetracycline, or benzalkonium chloride (preservative in Fucithalmic), simultaneous wearing of contact lenses, female patients of child bearing potential or those who were pregnant or breast feeding, concurrent use of prescribed anti-infective drugs, other ophthalmic complications, or severe renal impairment.

The drugs used in this study were Fucithalmic, a topical preparation of $1 \%$ fusidic acid in a carbomer gel made isotonic by adding mannitol, buffered to $\mathrm{pH} 5 \cdot 5$, and 
preserved with benzalkonium chloride; Fucithalmic placebo with similar gel but lacking the fusidic acid; oxytetracycline tablets containing $250 \mathrm{mg}$ oxytetracycline and oxytetracycline placebo - a matching tablet but lacking the oxytetracycline. Drugs were dispatched every 2 months to patients by the pharmacy so that they were unaware whether they were entering a placebo or active treatment phase.

The daily dosage for fusidic acid and placebo gel was one application of one drop of gel 12 hourly to the inferior fornix and to the eyelid margins, and for oxytetracycline and placebo tablets it was one tablet taken 12 hourly with $100 \mathrm{ml}$ of water, at least 1 hour before meals.

The maximum duration of therapy was 8 months, broken into four 2 month phases. All patients entering the study received topical placebo gel and placebo tablets for the first 2 month phase. In the following 2 month phase, a randomised $50 \%$ of patients received fusidic acid gel and oxytetracycline tablets, while the other $50 \%$ received either fusidic acid gel and placebo tablets or oxytetracycline tablets and placebo gel. This was followed by a third phase of 2 months in which all patients received placebo medication again. In the final 2 month phase, the $50 \%$ of patients who had originally received a combination of both fusidic acid and oxytetracycline were randomly selected so that equal numbers would receive either Fucithalmic gel and placebo tablets or oxytetracycline tablets and placebo gel. The remaining $50 \%$ of patients received a combination of both fusidic acid gel and oxytetracycline tablets.

The trial flow diagram, for each phase, is illustrated below:

\begin{tabular}{|c|c|c|}
\hline Placebo & (Phase 1) & Placebo \\
\hline $\begin{array}{l}\text { Fucithalmic }+ \\
\text { oxytetracycline }\end{array}$ & (Phase 2) & $\begin{array}{l}\text { Fucithalmic or } \\
\text { oxytetracycline }\end{array}$ \\
\hline Placebo & (Phase 3) & Placebo \\
\hline $\begin{array}{l}\text { Fucithalmic or } \\
\text { oxytetracycline }\end{array}$ & (Phase 4) & $\begin{array}{l}\text { Fucithalmic+ } \\
\text { oxytetracycline }\end{array}$ \\
\hline
\end{tabular}

Patient compliance was expected to be high, since blepharitis is a chronic disease and patients are usually well motivated to participate in treatment regimens. On entry to the study, inclusion criteria, based on previous assessment of symptoms and signs, ${ }^{2}$ and exclusion criteria were checked while demographic data, concurrent disease, and medication were recorded.

The following symptoms were recorded by the patients on diary score cards before the start of the trial and every 2 months thereafter, immediately before commencing the next treatment phase: burning, itching, grittiness, soreness, dryness, blurring of vision, pain, discharge, morning stickiness, redness, watering, photophobia, and aggravation of symptoms in smoky or hot environments. These symptoms were graded in severity; 0 absent, 1 mild, 2 moderate, and 3 severe.

The patients were examined by the investigators, at the start of the study and after each active treatment phase, for signs of chronic blepharitis including folliculitis, collarettes, eyelash loss, seborrhoea, telangectasia, meibomianitis, chronic inflammation of the lid margin, superficial punctate corneal epitheliopathy, and marginal keratitis. In addition, patients were examined for the presence of rosacea with excessive blushing, thickening and vascularisation of facial skin, rhinophyma, papular and pustular erythema, conjunctival hyperaemia and discharge, telangectasia of the lid margin, chalazia, and signs of keratitis. Lid microbiology was not performed in this study, as the aim was to determine the symptomatic response to each of the two antibiotic treatment regimens.

Data were analysed for the comparative efficacy of the three active treatment regimens (fucidin, oxytetracycline, or the combination) relative to the two placebo phases and to one another using Friedman's test, followed by the Mann Whitney U test, comparing magnitude of changes. This was based on the patients' subjective assessment of symptoms with each change of treatment and the investigators' assessment of signs, before and after treatment. The $\chi^{2}$ with Yates's correction was used to test differences in proportions (for example, responders/non-responders). All tests were two tailed and statistical significance was defined as $\mathrm{p}<0.05$.

\section{Results}

Sixty one patients with chronic blepharitis were recruited into this clinical trial of whom $43(71 \%)$ completed both placebo and an active treatment phase. Thirty four patients completed all four phases, seven completed three phases, and two completed two phases. Of 43 patients included in the analysis, 23 were male and 20 were female with a mean age of 56; three patients had unilateral blepharitis, the remainder being bilateral. Twenty seven patients were recruited from a previous study of chronic blepharitis, when 116 were tested for CMI to $S$ aureus, ${ }^{2}$ of whom 11 had given an enhanced CMI response. Sixteen additional patients were recruited with chronic blepharitis but were not tesisd for enhancement. No serious adverse reactions were reported.

Of 43 patients analysed, $18(44 \%)$ were thought to have concomitant non-ocular rosacea of mild degree (erythema and blushing of facial skin), one of moderate, and one of severe degree. Four patients were thought to have seborrhoea of mild degree. Nine patients $(23 \%)$ suffered from atopy, seven of mild, and two of moderate degree.

Analysis of symptoms, recorded by patients before the trial, gave an overall mean score of 23 (range 0 to 32) out of a possible 104 for both eyes together, with mean values of $22 \cdot 7$ and 22.6 respectively for those with and without enhanced CMI to $S$ aureus. Analysis of signs gave a mean score of 9.4 out of a possible 60 for each eye; mean scores for enhanced and non-enhanced patients were 10.3 and $9 \cdot 1$ respectively with no statistical difference. Within this patient group folliculitis was observed in only one patient. 
Table 1 Change in symptoms of blepharitis with change in treatment (placebo to active phase, self scored on diary cards)

\begin{tabular}{|c|c|c|c|c|}
\hline & $\begin{array}{l}\text { Improved } \\
\text { Ros/Non-R }\end{array}$ & $\begin{array}{l}\text { No change } \\
\text { Ros/Non-R }\end{array}$ & $\begin{array}{l}\text { Worse } \\
\text { Ros/Non-R }\end{array}$ & $\begin{array}{l}\text { \% Improvement } \\
\text { Ros/Non-R }\end{array}$ \\
\hline $\begin{array}{l}\text { Placebo to fusidic acid }(n=18) \\
\text { Placebo to oxytetracycline }(n=22) \\
\text { Placebo to fusidic acidt }\end{array}$ & $\begin{array}{l}6 / 0 \\
5 / 3\end{array}$ & $\begin{array}{l}1 / 1 \\
2 / 2\end{array}$ & $\begin{array}{l}1 / 9 \\
3 / 7\end{array}$ & $\begin{array}{l}75 / 0 \\
50 / 25\end{array}$ \\
\hline oxytetracycline $(n=34)$ & $6 / 5$ & $3 / 5$ & $8 / 7$ & $35 / 30$ \\
\hline
\end{tabular}

Ros=patient with rosacea Non $-\mathrm{R}=$ patient without rosacea

Initial analysis did not take account of the confounding factor of rosacea. Comparison of symptomatic responses to each antibiotic was not significant $(p=0 \cdot 8)$. Comparison of the magnitude of changes for fusidic acid plus oxytetracycline versus fusidic acid or oxytetracycline alone, and for fusidic acid versus oxytetracycline, was not significant. Comparison of 'matched pairs' in 30 patients who completed all four phases was performed for fusidic acid versus fusidic acid plus oxytetracycline for 12 patients, and for oxytetracycline versus fusidic acid plus oxytetracycline in 18 others, but no significant difference was found. Scoring of symptoms was also analysed for all patients known to have either enhanced or non-enhanced CMI to $S$ aureus, but no significant differences were found.

Symptoms were then analysed for patients with and without rosacea following active treatment (Table 1). Seventy five per cent of those with rosacea improved with fusidic acid alone $\left(\chi^{2}\right.$ test with Yates's correction $7 \cdot 6$, $\mathrm{p}<0.01), 50 \%$ with oxytetracycline, but only $35 \%$ with the combination. The reverse occurred in patients without rosacea; $90 \%$ were worse with fusidic acid alone but $25 \%$ improved with oxytetracycline.

The number of patients observed with improvement or no change at the end of each active treatment period compared with their own baseline, are given in Table 2 . Separate results are given for those patients with and without enhancement of CMI to $S$ aureus; 19 of 20 treatment episodes in 11 patients with enhancement gave improvement (or no change) on antibiotic therapy compared with 23 of 30 treatment episodes in 16 patients without enhancement. This was not statistically significant.

\section{Discussion}

Open, randomised, comparative clinical trials are often employed when comparing the efficacy of different antibacterial treatments for example, in conjunctivitis. ${ }^{14}$ However, in chronic conditions, such as blepharitis in

Table 2 Analysis of signs in blepharitis (placebo to active phase, scored by ophthalmologist)

\begin{tabular}{llll}
\hline & \multicolumn{4}{l}{ Improved or no change/worse (\% better or no change) } \\
\cline { 2 - 4 } & $\begin{array}{l}\text { Placebo to } \\
\text { fusidic acid }\end{array}$ & $\begin{array}{l}\text { Placebo to } \\
\text { oxytet }\end{array}$ & $\begin{array}{l}\text { Placebo to fusidic } \\
\text { acid }+ \text { oxytet }\end{array}$ \\
\hline Non-enhanced $(\mathrm{n}=16)$ & $5 / 2$ & $6 / 2$ & $12 / 3(77)$ \\
CMI enhanced $(\mathrm{n}=11)^{\star}$ & $5 / 0$ & $5 / 0$ & $9 / 1(95)$ \\
CMI not known $(\mathrm{n}=16) \dagger$ & $4 / 0$ & $5 / 1$ & $9 / 1(90)$ \\
\hline
\end{tabular}

$\star 8 / 11$ had rosacea; $\uparrow 40 \%$ expected to have enhanced CMI for $S$ aureus $^{2}$.

$\mathrm{CMI}=$ cell mediated immunity; oxytet $=$ oxytetracycline. which the aetiology is unproved, and the response frequently assessed on symptomatic grounds, a placebo controlled study is required to establish efficacy of antibacterial therapy. Furthermore, a double blind design is essential to remove the possibility of both investigator and patient bias.

This study which was double blind and placebo controlled required each patient to receive placebo treatment for a 2 month period before each of two active treatment phases, when changes in symptoms and signs were investigated. The study design also allowed for crossover between combination and single drug therapy and should be a useful model for future trials of drug therapy in blepharitis. A third 2 month period of placebo treatment at the end of the trial would have been useful, as it would have allowed an evaluation of the effect of withdrawing active therapy and the detection of any relapse that occurred. The use of a self assessment scheme removed the bias that can occur with interrogation by an investigator.

Forty seven per cent of patients in this trial suffered from rosacea, compared with $27 \%$ in the previous study. ${ }^{2}$ This was unexpected but has allowed investigation of therapy for rosacea associated blepharitis within a randomised, controlled trial. Analysis of the 20 patients with rosacea has shown that topical fusidic acid alone was most beneficial in relieving symptoms $(75 \%)$. This may reflect recently described in vitro immunosuppressive effects of fusidic acid which inhibited $\mathrm{T}$ cell proliferation similarly to cyclosporin A. ${ }^{15}$

Results for oxytetracycline in the rosacea group are similar to those of Bartholomew who found that it produced a higher number of remissions (19 of 35) compared with the group given placebo (11 of 35). ${ }^{11}$ These authors demonstrated that non-specific signs, including blepharitis, responded well to oxytetracycline but found no permanent change in conjunctival and corneal vascularisation. Their findings of a high remission rate in one third of their patients using placebo demonstrates that spontaneous remissions can occur. Others have found comparable results in rosacea of skin without ophthalmic signs when treating with oral and topical metronidazole in double blind placebo controlled trials ${ }^{16}$; the explanation is unknown but suppression of cell mediated immunity has been suggested. In addition, one other controlled trial of papular rosacea of the skin demonstrated a comparable effect between oxytetracycline and ampicillin, both significantly better than placebo. ${ }^{12}$ Doxycycline has been shown to be beneficial in ocular rosacea, as a once daily dose, but the study was open and uncontrolled. ${ }^{13}$ These controlled studies, and our own patients, confirm the uncontrolled findings of Brown ${ }^{17} 18$ that oral oxytetracycline gave symptomatic improvement in ocular rosacea patients although half relapsed on drug withdrawal.

An association between ocular rosacea and lid infection with $S$ aureus, including blepharitis, marginal ulceration, and other corneal complications, has been pursued for 50 years. ${ }^{19}$ Brown demonstrated succinctly that 
lid colonisation with $S$ aureus often occurred in patients with rosacea. They found that such colonisation did not change with oxytetracycline treatment on a qualitative basis although they did not investigate quantitatively. ${ }^{17} 18$ Lempert ${ }^{20}$ has shown that chalazia of the lids are more common in rosacea (64\%) compared with a control group (13\%). Others have described granulomatous disease of the lids in rosacea in both the black ${ }^{21}$ and white population, ${ }^{22}$ the latter suggesting similarities of histological lesions with mycobacterial and syphilitic disease. We have observed in this study that eight out of 11 patients with enhanced CMI to $S$ aureus has rosacea. This observation is worthy of further study.

The relation between rosacea and CMI enhancement to $S$ aureus remains speculative but both appear frequently to coexist; in the previous study this enhancement was associated with folliculitis. ${ }^{2}$ In addition, rosacea lids are frequently colonised with $S$ aureus, in a similar manner to the atopic individual but in those in which this CMI is not enhanced. ${ }^{3}$ While it is not understood why patients with rosacea and others develop CMI enhancement to $S$ aureus, the continued presence of $S$ aureus on the lid margin of the enhanced patient can be expected to contribute to a chronic inflammatory reaction of granulomatous type. This, in turn, would benefit from both the antiinflammatory effect of oxytetracycline (and possibly fusidic acid and metronidazole) as well as the antibacterial effect of topical fusidic acid which reduces $S$ aureus colonisation of lids. Failure of systemic oxytetracycline to eradicate lid colonisation by $S$ aureus in rosacea ${ }^{17} 18$ is expected. The observation that the combination of fusidic acid and oxytetracycline was less frequently effective than either alone was surprising (Table 1). It was not statistically significant, however, and warrants further investigation particularly with reference to therapy for systemic symptoms.

Patients with blepharitis but without rosacea did not benefit symptomatically from topical fusidic acid and there was less benefit from oxytetracycline. This reflects the different aetiologies of blepharitis in these patients. Coagulase negative staphylococci were unlikely to have been pathogenic in either group, based on our previous work. ${ }^{24}$ Treatment of blepharitis has in the past been based on laboratory tests demonstrating oxytetracycline inhibition of lipase production by coagulase negative staphylococci. ${ }^{10}$ Suppression of this bacterial enzyme in meibomianitis ${ }^{10}$ is still theoretical and controlled clinical trials need to be performed.

In conclusion, we have found that symptoms of rosacea associated blepharitis frequently responded well to topical fusidic acid gel as well as to systemic oxytetracycline but responded less well to the combination. In contrast, symptoms of other patients with blepharitis did not respond to topical fusidic acid. Analysis of signs has suggested greater improvement with fusidic acid or oxytetracycline in those patients with enhancement to $S$ aureus, although this was not proved statistically. This suggests that before commencing treatment for blepharitis, it should be established whether there is coexisting rosacea, particularly mild and limited to excessive blushing, and treatment targeted accordingly.

This trial was conducted under clinical trials exemption and was approved by Moorfields Hospital ethics committee. The study was conducted according to the Declaration of Helsinki study was conducted according to the Declaration of Helsinki as adopted by the 18th World Medical Assembly (1965) and as
revised by the World Medical Assemblies Tokyo (1975) and revised by the
Venice (1983).

We are grateful to $\mathrm{Mr} \mathrm{V}$ Andrews, principal pharmacist, Moorfields Eye Hospital for distributing placebo and active treatments to our patients on a randomised basis, and to Mr I A Mackie for advice.

1 McCulley JP, Dougherty JM, Deneau DG. Classification of chronic blepharitis. Ophthalmology 1982; 89: 1173-80.

2 Ficker L, Ramakirshman M, Seal DV, Wright P. Role of cell-mediated immunity to staphylococci in blepharitis. Am $\mathcal{f}$ Ophthalmol 1991; 111: 473-9.

3 Ficker L, Seal DV, Wright P. Staphylococcal blepharitis (Chapter 58). In: Pepose J, Holland G, Wilhelmus K, eds. Ocular infection and immunity. Chicago: Mosby, 1994.

4 Seal D, Ficker L, Wright P. Role of coagulase-negative staphylococci in chronic blepharitis. Microb Ecol Health Dis 1992; 5: 69-75

5 Hvidberg J. Fusidic acid in acute conjunctivitis. Acta Ophthalmol 1987; 65: 43-7.

6 Dirsdal $M$. Fucithalmic in acute conjunctivitis. Acta Ophthalmol 1987; 65: 129-33.

7 Taylor B, Burd EM, Tabbara KF. Corneal and intra-ocular penetration of topical and subconjunctival fusidic acid. Br $\mathcal{F}$ Ophthalmol 1987; 71: 598-601.

8 Taylor PB, Tabbara KF, Burd EM. Effect of preoperative fusidic acid on the normal eyelid and conjunctival bacterial flora. $\mathrm{Br} \mathcal{F}$ Ophthalmol 1988; 72: 206-9.

9 Dilly PN, Mackie IA. Distribution of tetracycline in the conjunctiva of patients on long-term systemic doses. Br f Ophthalmol 1981; 69: 25-8.

10 Dougherty JM, McCulley JP, Silvany ME, Meyer DR. The role of tetracycline in chronic blepharitis. Invest Ophthalmol Vis Sci 1991; 32: 2970-5.

11 Bartholomew RS, Reid BJ, Cheesborough MJ, Macdonald $M$, Galloway NR. Oxytetracycline in the treatment of ocular rosacea: a double-blind trial. Br f Ophthalmol 1982; 66: $386-8$.

12 Marks $R$, Ellis J. Comparative effectiveness of oxytetracycline and ampicillin in rosacea. Lancet 1971; ii: 1049-52.

13 Frucht-Pery J, Chayet AS, Feldman ST, Lin S, Brown SI. The effect of doxycycline on ocular rosacea. Am $\dot{f}$ Ophthalmol 1989; 107: 434-5.

14 Trimethoprim-Polymyxin B Sulphate Ophthalmic Ointment Study Group. Trimethoprim-polymyxin B sulphate ointment versus chloramphenicol ophthalmic ointment in the treatment of bacterial conjunctivitis. f Antimicrob Chemother 1989; 23: 261-6.

15 Bendtzen K, Diamant M, Faber V. Fusidic acid, an immunosuppressive drug with functions similar to immunosuppressive drug with function

16 Nielsen PG. Treatment of rosacea with $1 \%$ metronidazole cream. A double-blind study. Br $\mathcal{f}$ Dermatol 1983; 108: 327-32.

17 Brown S, Shahinian L. Diagnosis and treatment of ocular rosacea. Ophthalmology 1978; 85: 779-86.

18 Jenkins MS, Brown SI, Lempert SL, Weinberg RJ. Ocular rosacea. Am f Ophthalmol 1979; 88: 618-22.

19 Wise G. Ocular rosacea. Am $\mathcal{F}$ Ophthalmol 1943; 26: 591-609.

20 Lempert SL, Jenkins MS, Brown SI. Chalazia and rosacea. Arch Ophthalmol 1979; 97: 1652-3.

21 Browning DJ, Rosenwasser G, Lugo M. Ocular rosacea in Browning DJ, Rosenwasser G, Lugo M. Ocu
blacks. Am f Ophthalmol 1986; 101: 441-4.

22 Patrinely JR, Font RL, Anderson RL. Granulomatous acne rosacea of the eyelids. Arch Ophthalmol 1990; 108: $561-3$ 